\title{
CRITICAL BEHAVIOUR OF SOME 2D LATTICE MODELS
}

\author{
G. Kamieniarz ${ }^{b *}$, H.W.J. Blöte ${ }^{a}$ AND R. DekeyseR ${ }^{b}$ \\ a Faculty of Applied Physics, Delft University of Technology, The Netherlands \\ ${ }^{b}$ Institute of Theoretical Physics, KU Leuven, Belgium
}

The density series of the non-interacting hard-square lattice gas model are reanalyzed by the ratio, Dlog Padé and differential approximant methods. The problem of poor consistency between series and other results is resolved. Transfer matrix calculations are performed, implementing both finite-size scaling and conformal invariance. Very accurate estimates of the critical exponents $y_{t}$ and $y_{h}$ are obtained in agreement with Ising universality. Furthermore, an improvement of the value of the critical density $\rho_{c}$ is found. In addition, the universal critical-point ratios of the square of the second and the fourth moment of the magnetization for ferromagnetic Ising models on the square and on the triangular lattice with periodic boundary conditions are reported.

PACS numbers: 05.50.+q, 64.60.-i, 64.60.Cn, 64.60.Ak, 64.60.Fr, 75.40.Mg

\section{Introduction}

For the non-interacting hard-square model [1], the absence or presence of a lattice-gas particle at site $i$ is expressed by a variable $\sigma_{i}=0$ or $\sigma_{i}=1$, respectively. Denoting the activity of the gas particles as $z$, the partition sum is

$$
Z=\sum_{\sigma_{i}} z^{\sum_{i} \sigma_{i}} \prod_{\langle i, j\rangle}\left(1-\sigma_{i} \sigma_{j}\right) .
$$

The product is over all pairs of nearest-neighbour sites, and guarantees that configurations with interpenetrating particles do not contribute to $Z$.

In absence of an exact solution, Baxter et al. applied series expansion techniques to determine the critical exponents of this model. They found that the specific heat exponent $\alpha^{\prime}=0.09 \pm 0.05$ which was different from the exactly known value of the two-dimensional Ising model $\alpha=0$. Although subsequent analyses of the finite-size scaling behaviour of the temperature derivative of the correlation length $[2,3]$ did not show significant deviations from Ising universality, we found the result by Baxter et al. sufficiently challenging to find more compelling numerical evidence that the hard-square model is inside the Ising universality class.

\footnotetext{
*Permanent address: Institute of Physics, A. Mickiewicz University, Poznań, Poland.
} 


\section{Analysis of the high-density series}

The value of the critical activity $z_{c}$ [4], where the translational symmetry of the model (1) is spontaneously broken, is $z_{\mathrm{c}}=3.796255174(3)$. On the basis of this result for the critical activity $z_{\mathrm{c}}$, the series expansions evaluated by Baxter et al. can be reanalyzed.

Thus we consider an expansion of the order parameter (i.e. the staggered density) $R$ in terms of the high-density parameter $x=1 / z$. From the ratio analysis of $R^{-8}(x)$, using $z_{\mathrm{c}}$ and the coefficients listed in Table II of [1] we have estimated that the critical exponent $\beta=0.1249(1)$, in agreement with the Ising universality.

The subsequent Dlog Padé analysis of the series $R(x)$ (as that in Table I of [1]) and the smooth dependence of the residues of $\frac{\mathrm{d}}{\mathrm{dx}} \ln R(x)$ on the distance of the corresponding poles from the critical value $x_{c}=0.263417488$ indicate that $\beta$ does not differ from $1 / 8$ by more than a few times $10^{-5}$.

To calculate $\alpha$, we choose the second derivative of the lattice-gas density series $\rho$ expressed in terms of $x$ :

$$
\rho^{(2)} \equiv \frac{\mathrm{d}^{2}}{\mathrm{~d} x^{2}} \rho^{\prime} \sim\left(x_{c}-x\right)^{-1-\alpha^{\prime}},
$$

which is more singular than $\rho$ and can be found from the series $\rho^{\prime}=1-2 \rho=$ $\sum_{n=1}^{\infty} \rho_{n} x^{n}$, where the coefficients $\rho_{n}$ are determined by those given in Appendix B of Ref. [1]. Performing the Dlog analysis of the $\rho^{(2)}$ series, we estimate $\alpha^{\prime}=-0.1(1)$ which indicates that $\alpha^{\prime}$ may be smaller than the result obtained by Baxter et al. from the $R\left(\rho^{\prime}\right)$ series.

TABLE I

The differential approximant analysis of the series $R^{-1}\left(\rho^{\prime}\right)$.

\begin{tabular}{c|c|c|c|c|c}
\hline \hline$N$ & $L$ & $M$ & $\bar{\rho}_{\mathrm{c}}^{\prime}$ & $\left(\frac{\beta}{1-\alpha^{\prime}}\right)_{\mathrm{u}}$ & $\left(\frac{\beta}{1-\alpha^{\prime}}\right)_{\mathrm{b}}$ \\
\hline 7 & 7 & 7 & 0.264465 & 0.118388 & 0.126657 \\
8 & 5 & 8 & 0.264488 & 0.122358 & 0.126509 \\
7 & 6 & 8 & 0.264483 & 0.121542 & 0.126559 \\
8 & 4 & 9 & 0.264535 & 0.129885 & 0.126066 \\
6 & 7 & 8 & 0.263892 & 0.018301 & 0.126448 \\
7 & 5 & 9 & 0.264491 & 0.122845 & 0.126751 \\
5 & 8 & 8 & 0.264692 & 0.157027 & 0.125629 \\
6 & 6 & 9 & 0.264552 & 0.132852 & 0.126476 \\
5 & 7 & 9 & 0.264583 & 0.137947 & 0.126610 \\
6 & 5 & 10 & 0.264471 & 0.119259 & 0.145598 \\
4 & 8 & 9 & 0.264060 & 0.041619 & 0.123054 \\
5 & 6 & 10 & 0.264597 & 0.140181 & 0.124088
\end{tabular}


TABLE II

The differential approximant analysis of the series $\rho(x)$

\begin{tabular}{c|c|c|c|c|c}
\hline \hline$N$ & $L$ & $M$ & $\overline{x_{\mathrm{c}}}$ & $\left(1-\alpha^{\prime}\right)_{\mathrm{u}}$ & $\left(1-\alpha^{\prime}\right)_{\mathrm{b}}$ \\
\hline 7 & 6 & 7 & 0.263490 & 0.985605 & 0.999678 \\
8 & 4 & 8 & 0.263516 & 0.981007 & 0.994742 \\
6 & 7 & 7 & 0.263417 & 0.999936 & 0.999765 \\
7 & 5 & 8 & 0.263608 & 0.963034 & 0.998233 \\
6 & 6 & 8 & 0.263422 & 0.998815 & 0.999693 \\
7 & 4 & 9 & 0.263491 & 0.984604 & 0.995466 \\
5 & 7 & 8 & 0.263417 & 0.999779 & 0.999766 \\
6 & 5 & 9 & 0.263549 & 0.973867 & 0.100050 \\
4 & 8 & 8 & 0.263415 & 0.999955 & 0.999424 \\
5 & 6 & 9 & 0.263494 & 0.984681 & 0.999637 \\
4 & 7 & 9 & 0.263330 & 1.017277 & 1.000238 \\
5 & 5 & 10 & 0.263543 & 0.975050 & 0.995529
\end{tabular}

In Tables I and II our additional differential approximant analyses [5] of $R^{-1}\left(\rho^{\prime}\right)$ and $\rho(x)$ series are shown. The columns denoted $N, L, M$ display the degrees of the corresponding polynomials. $\overline{\rho_{\mathrm{c}}^{\prime}}$ and $\overline{x_{\mathrm{c}}}$ represent the calculated unbiased critical parameters $\rho_{\mathrm{c}}^{\prime}$ and $x_{\mathrm{c}}$ which are obtained from the given approximants. Subscripts $u$ and $\mathrm{b}$ denote the unbiased and biased (choosing $\rho_{\mathrm{c}}^{\prime}=0.264514$, $\left.x_{\mathrm{c}}=0.2634175\right)$ estimates the corresponding critical exponents. The accuracy of the u-exponents depends strongly on the accuracy of the estimates $\overline{\rho_{\mathrm{c}}^{\prime}}$ and $\overline{x_{\mathrm{c}}}$. However, the b-exponents deviate from the Ising values within a margin of 0.002 and 0.005 for $\beta /\left(1-\alpha^{\prime}\right)$ and $\alpha^{\prime}$, respectively (where we disregard the estimate for the approximant $[6,5])$.

\section{Finite-size-scaling analysis}

We consider the model (1) on $L \times L$ and $L \times \infty$ square lattices with periodic boundary conditions. To enable the introduction of two sublattices in a checkerboard-like fashion, $L$ is restricted to be even. We use the transfer matrix technique in order to calculate the partition sum (1), as well as some of its derivatives. Denoting the lattice gas variables $\left(\sigma_{j 1}, \sigma_{j 2}, \cdots, \sigma_{j L}\right)$ in the $j$-th row of the lattice by $\sigma_{j}$, the elements of the row-to-row transfer matrix $\boldsymbol{T}$ are

$$
T\left(\sigma_{i}, \sigma_{j}\right)=x^{\frac{\mu}{2} \sum_{k=1}^{L}\left(\sigma_{i, k}+\sigma_{j, k}\right)} \prod_{k=1}^{L}\left(1-\sigma_{i, k} \sigma_{j, k}\right)\left(1-\sigma_{i, k-1} \sigma_{i, k}\right)\left(1-\sigma_{j, k-1} \sigma_{j, k}\right)
$$

with $\sigma_{i, 0}=\sigma_{i, L}$ in accordance with the periodic boundary conditions. The partition sum (1) of a system consisting of $M$ rows is equal to $Z=\operatorname{Tr} T^{M}$. For further details of the numerical methods, see e.g. [6]. 
A finite-size analysis can be used to determine $y_{h}$ and $y_{t}$. Other exponents follow from these by means of scaling. Here the main interest is in the value of $y_{t}$ in view of its relevance for $\alpha$ expressed by $\alpha=\left(d-y_{t}\right) / y_{t}$. The assumptions involved amount to the analyticity of renormalization transformations employing an additional finite-size field [7] $1 / L$ with exponent $y_{L}=1$.

We have determined finite-size data for several quantities: the density $\rho_{L}$ of the lattice gas particles, the specific heat $c_{L}$, the staggered susceptibility $\chi_{L}$, and the temperature derivative $g_{i}^{\prime}(L)$ of the inverse magnetic correlation length. They are expected to fulfil the finite-size scaling relations

$$
\begin{array}{ll}
\rho_{L} \approx \rho_{\mathrm{c}}+L^{y_{t}-d}\left(a_{1}+a_{2} L^{-p}\right), & c_{L} \approx L^{2 y_{t}-d}\left(b_{1}+b_{2} L^{-q}\right), \\
\chi_{L} \approx L^{2 y_{h}-d}\left(c_{1}+c_{2} L^{-r}\right), & g_{i}^{\prime}(L) \approx L^{y_{t}-1}\left(d_{1}+d_{2} L^{-w}\right),
\end{array}
$$

where $d=2$ is the dimensionality and the terms with exponents $p, r, q, w$ represent corrections to scaling. As an example, we show the results for a square system in Table III. The scaling behaviour of all these data is in accurate agreement with the Ising universal exponents.

TABLE III

The finite-size data $2 \rho_{L}, c_{L}$ and $\chi_{L}$ calculated numerically for square systems.

\begin{tabular}{c|c|c|c}
\hline \hline$L$ & $2 \rho_{L}$ & $c_{L}$ & $\chi_{L}$ \\
\hline 2 & 0.8090898056421140 & 0.1402349991566989 & 0.724743813172343 \\
4 & 0.7749712801688777 & 0.1922474459514767 & 2.447647203710980 \\
6 & 0.7616725216302425 & 0.2246002865877822 & 4.978297322277479 \\
8 & 0.7550639427551426 & 0.2477057485415912 & 8.241664994051973 \\
10 & 0.7511214217544006 & 0.2656570381718074 & 12.18609189883877 \\
12 & 0.7485021560614531 & 0.2803252992432338 & 16.77381425559746 \\
14 & 0.7466353591805462 & 0.2927219631820310 & 21.97568172323616 \\
16 & 0.7452373256688473 & 0.3034545757008545 & 27.76817664548804
\end{tabular}

Another approach intimately related with renormalization was made using the hypothesis of conformal invariance [8]. The temperature and magnetic exponents were determined using the relation between exponents and the finite-size amplitude of the associated correlation lengths for the infinite strips. The ensuing results

$$
y_{t}=1.000000(1), \quad y_{h}=1.875000(1)
$$

are in precise agreement with the exactly known Ising values $y_{h}=15 / 8$ and $y_{t}=1$. density

In addition to these results for the exponents, we have also found the critical

$$
2 \times \rho_{\mathrm{c}}=0.73548600(1)
$$

and the universal critical point ratio

$$
Q=\left\langle R^{2}\right\rangle^{2} /\left\langle R^{4}\right\rangle=0.85625(5)
$$


the latter in agreement with the corresponding Ising value we have recently determined [6]

$$
Q=0.856216(1) \text { and } Q=0.85872528(3)
$$

for ferromagnetic Ising models with periodic boundaries of square and hexagonal symmetries, respectively.

Summarizing, the whole body of numerical results provides strong evidence that the non-interacting hard-square model belongs to the Ising universality class, in agreement with the topology of the phase diagram.

\section{References}

[1] R.J. Baxter, I.G. Enting, S.K. Tsang, J. Stat. Phys. 22, 465 (1980).

[2] D.W. Wood, M. Goldfinch, J. Phys. A, Math. Gen. 13, 2781 (1980).

[3] Z. Racz, Phys. Rev. B 21, 4012 (1980).

[4] H.W.J. Blöte, X.N. Wu, J. Phys. A, Math. Gen. 23, L627 (1990).

[5] M.E. Fisher, H. Au-Yang, J. Phys. A, Math. Gen. 12, 1677 (1979).

[6] G. Kamieniarz, H.W.J. Blöte, J. Phys. A, Math. Gen. 26, 201 (1993).

[7] M.N. Barber, in: Phase Transitions and Critical Phenomena, Vol. 8, Eds. C. Domb, J.L. Lebowitz, Academic Press, London 1983, p. 145.

[8] J.L. Cardy, in: Phase Transitions and Critical Phenomena, Vol. 11, Eds. C. Domb, J.L. Lebowitz, Academic Press, New York 1987, p. 55. 